\title{
The study of the numerical diffusion in computational calculation
}

\author{
Kamila Kotrasova ${ }^{1, *}$, and Vladimira Michalcova ${ }^{2}$ \\ ${ }^{1}$ Department of Structural Mechanics, Institute of Structural Engineering, \\ Faculty of Civil Engineering, The Technical University of Kosice, 042 00Kosice, Slovak Republic \\ ${ }^{2}$ Department of Structural Mechanics, Faculty of Civil Engineering, \\ VŠB - Technical University of Ostrava, 70833 Ostrava-Poruba, Czech Republic
}

\begin{abstract}
The numerical simulation of flow process and heat transfer phenomena demands the solution of continuous differential equation and energy-conservation equations coupled with the continuity equation. The choosing of computation parameters in numerical simulation of computation domain have influence on accuracy of obtained results. The choose parameters, as mesh density, mesh type and computation procedures, for the numerical diffusion of computation domain were analysed and compared. The CFD simulation in ANSYS - Fluent was used for numerical simulation of 3D stational temperature flow of the computation domain.
\end{abstract}

\section{Introduction}

Computational Fluid Dynamics (CFD) is a branch of mechanics of continuum that uses numerical analysis and data structures to solve and analyse problems that involve flows of fluid, gas [1-3].

CFD is a tool with amazing flexibility, accuracy and breadth of application [4]. But serious CFD, the kind that provides insights to help you optimize your designs, can be out of reach unless you choose your software carefully [5-7]. To get serious CFD results, you need serious software. ANSYS CFD goes beyond qualitative results to deliver accurate quantitative predictions of fluid interactions and trade-offs $[7,8]$.

The software ANSYS-Fluent is used the discretization process for solution of transport equations [9-15]. The basic problem of transport equations solution the exact calculation of the transport variable $\Phi$ over the walls of a specific volume and its convective flow across these boundaries [16-22]. If the problem is computing, it is necessary to reckon on by computing with on the occurrence of the so-called "false" numerical diffusion and also on the occurrence of transport variable values $\Phi$ that are outside the scope of the correct solution [23,24]. This paper deals about the comparing of the physical accuracy of the calculation using the discretionary computational schemes proposed in the CFD code of the ANSYS-Fluent software and deals with solution how to reduce these numerical errors [2532].

\footnotetext{
*Corresponding author: kamila.kotrasova@tuke.sk
} 


\section{Numerical experiments, evaluation}

The aim of the numerical experiments was the evaluation of the physical accuracy of the numerical calculation in depending on the choice simulation parameters as: the choice of grid mesh density, the choice of grid mesh type and the choice of computation procedure.

A 3D stationary flow of fictitious gas with density $\rho=1 \mathrm{kgm}^{-3}$ was numerical simulated in the computation domain with dimension $1 \times 1 \times 0.2$ meters, see Fig. 1 . The gas thermal conductivity $\lambda\left[\mathrm{Wm}^{-1} \mathrm{~K}^{-1}\right]$ and the gas dynamic viscosity $\mu[\mathrm{Pa} \cdot \mathrm{s}]$ were approaching zero. The temperature field of computing domain was monitored. In this case, the transport variable $\Phi$ is the temperature of the fictitious gas.

The boundary conditions are considered:

- entry into the computation domain:

$0 \quad$ the inlet of velocity is constant on the left and up walls, the velocity vectors were considered $v_{x}=5 \mathrm{~ms}^{-1}$ and $v_{y}=5 \mathrm{~ms}^{-1}$,

0 the temperature:

- left wall was considered $400 \mathrm{~K}$,

- up wall was considered $300 \mathrm{~K}$,

- output from the computation domain: the outlet of pressure was given $p=0 \mathrm{~Pa}$,

- $\quad$ the side walls of computation domain were symmetry,

$\circ$ the all flow quantities across the boundary were zero,

$\circ$ the normal velocity was zero.

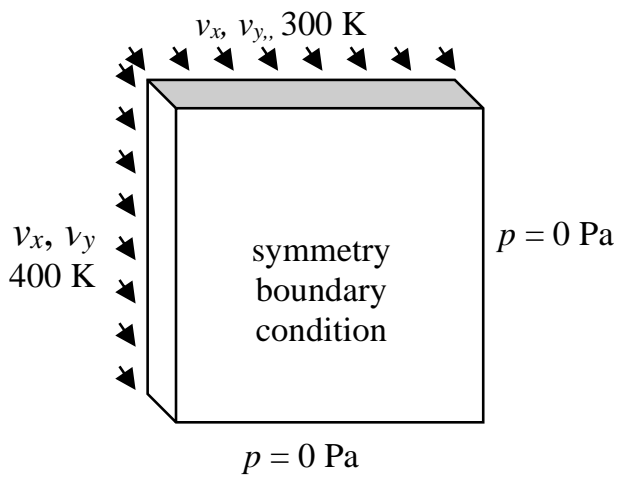

Fig. 1. The computation domain of numerical experiment.

a)

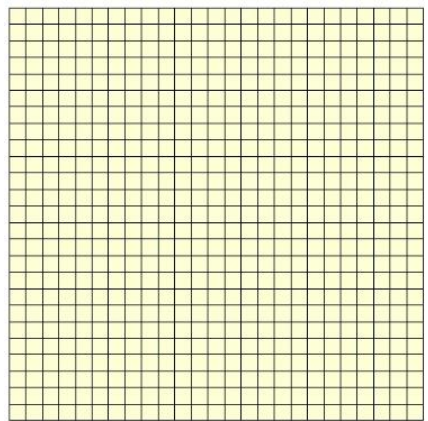

b)

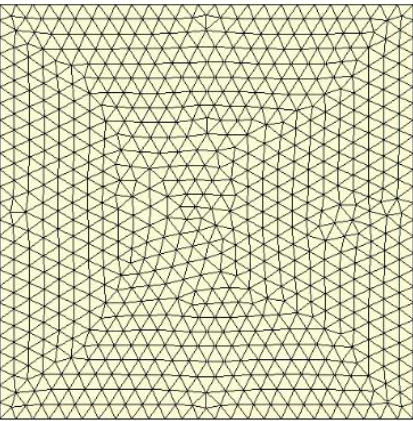

c)

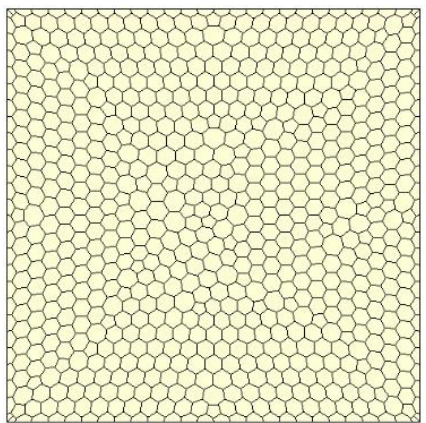

Fig. 2. Detail of grid mesh types, a) Hexahedra, b) Tetrahedra, c) Polyhedra. 
The object of the exploration was to determine the temperature variance (numerical diffusion) of the temperature field and to evaluate the temperature values, which are outside the range of the considered boundary conditions (out of the range $300 \mathrm{~K}-400 \mathrm{~K}$ ) with different choosing of computation parameters.

The numerical experiments were solved by considering of three typologies of grid (Hexahedra, Tetrahedra and Polyhedra) whose shapes are shown in Fig. 2. Hexahedra and Tetrahedra cells were made of 40 or 100 mesh parameters considering on the length of $1 \mathrm{~m}$ on all edges of the area in longitudinal section. The Polyhedra cells were generated in the ANSYS-Fluent software from the Tetrahedra cells. We become the total 6 computational areas of the same dimensions $1 \times 1 \times 0.2$ meters with different number of cells. The numbers of cells are for typology of grid:

- Hexahedra

- for 40 mesh parameter -12800 cells,

○ for 100 mesh parameter - 200000 cells,

- Tetrahedra

○ for 40 mesh parameter -83900 cells,

○ for 100 mesh parameter - 1332000 cells,

- Polyhedra

- for 40 mesh parameter -16600 cells,

○ for 100 mesh parameter -238000 cells.

The computing with Tetrahedra and Polyhedra cells were tested with three computational procedure:

- $\quad$ First-order upwind,

- Second-order upwind,

- Third-order Muscl.

For Hexahedra cells, the calculation was also performed with the Quick computational procedure too.

\section{Evaluation of numerical results}

The figures in chapter 3 represent the temperature field in longitudinal sections through the centre of the computational computation domains by considering of different computation conditions. The temperature scale in kelvin $[\mathrm{K}]$ in Figs. 4, 5 are in interval from $300 \mathrm{~K}$ to $400 \mathrm{~K}$ and it is valid for Figs. 3, 4, 5. Ideally, the temperature field should be diagonally sharply divided into two fields, corresponding to the input parameters. The temperatures should be in the interval $300 \mathrm{~K}-400 \mathrm{~K}$. The influence of grid mesh density, grid mesh type, the calculation scheme and computation procedure (a method of solving the gradient of a transport variable) was studied.

\subsection{Choosing of grid mesh density}

The grid mesh density has a significant influence on the size of the numerical diffusion. Fig. 3 illustrates the temperature fields for the Hexahedra grid mesh type in depending on the Hexahedra grid mesh for 40 and 100 meshes per length $1 \mathrm{~m}$ by using a First order and Second-order calculation procedures. The choice of calculation scheme is clear.

\subsection{Choosing of grid mesh type}

The grid mesh type has also influence on the numerical diffusion of the transport variable. The influence of grid mesh type on the numerical diffusion is not so significant. Fig. 4. 
presents the temperature fields for all three-grid mesh type (Hexahedra mesh cells, Tetrahedra mesh cells, Polyhedra mesh cells) of grid mesh density (100 meshes per length 1 $\mathrm{m})$ for Second-order calculation procedure.

a)

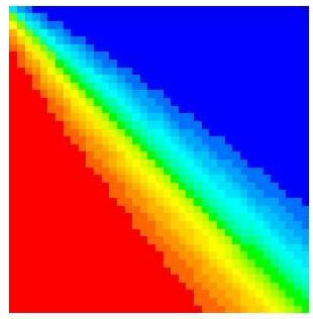

b)

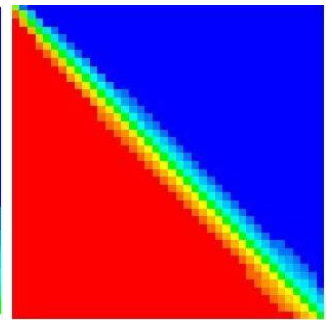

c)

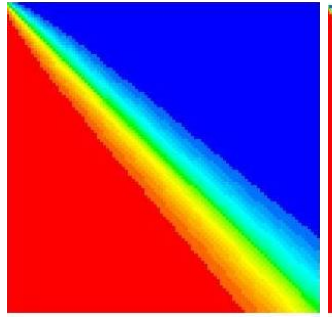

d)

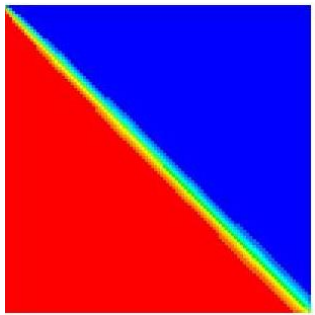

Fig. 3. The diffusion of the temperature field for Hexahedra mesh type, a) mesh $40 / \mathrm{m}$, First-order calculation scheme, b) mesh 40/m, Second-order calculation scheme, c) mesh 100/m, First-order calculation scheme, d) mesh 100/m, Second -order calculation scheme.

a)

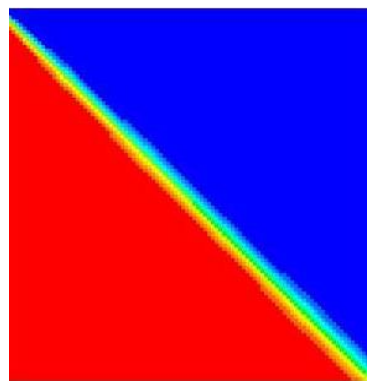

b)

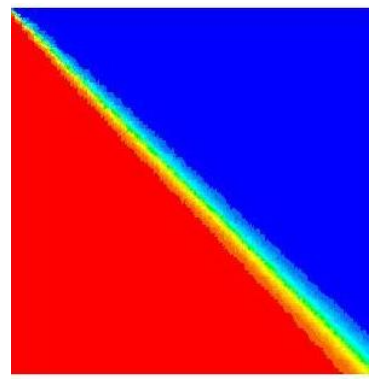

c)
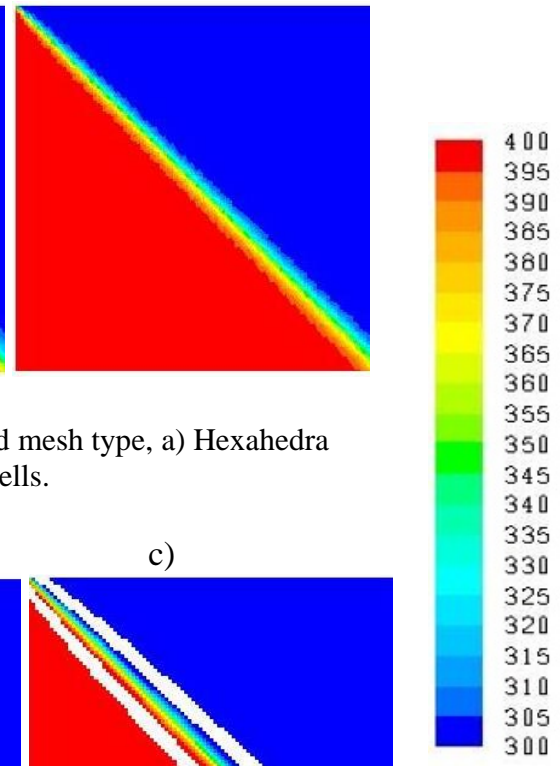

Fig. 4. Diffusion of the temperature field, influence of grid mesh type, a) Hexahedra mesh cells, b) Tetrahedra mesh cells, c) Polyhedra mesh cells.

a)

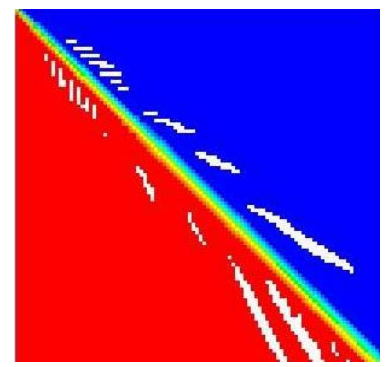

b)

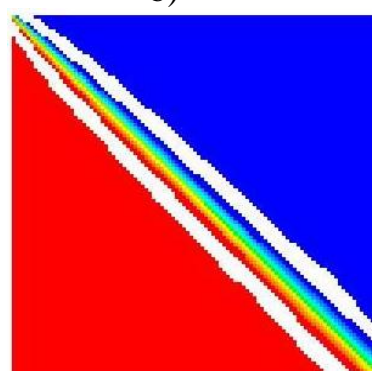

c)

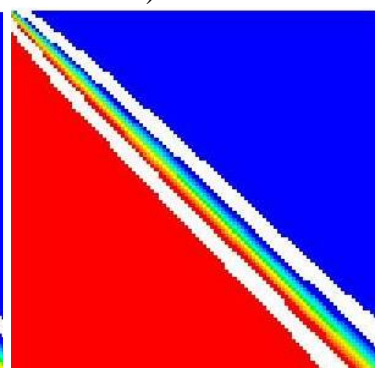

00

395

(390

(80

375

370

365

360

355

350

345

330

325

320

15

310

300

Fig. 5. Diffusion of the temperature field and the occurrence of values outside the input data range, influence of calculation scheme for Hexahedra grid mesh type, a) Quick calculation scheme,

b) Second-order scheme, c) Third-order Muscl calculation scheme.

\subsection{Choosing of calculation scheme}

The computation procedure has influence on the numerical diffusion and on occurrence of values outside of the input parameters. The values outside of the input parameters is seen as 
white colour areas in Fig. 5. The temperature fields for grid mesh density of 100 meshes per length $1 \mathrm{~m}$ by using of the Hexahedra grid mesh type for three computation procedure: a) Quick calculation procedure, b) Second -order procedure, c) Third-order Muscl calculation procedure. Using of the Quick calculation procedure in the numerical diffusion of gas domain gives the most accurate results and the smallest occurrence of values outside the input data range, see Fig. 5.

a)

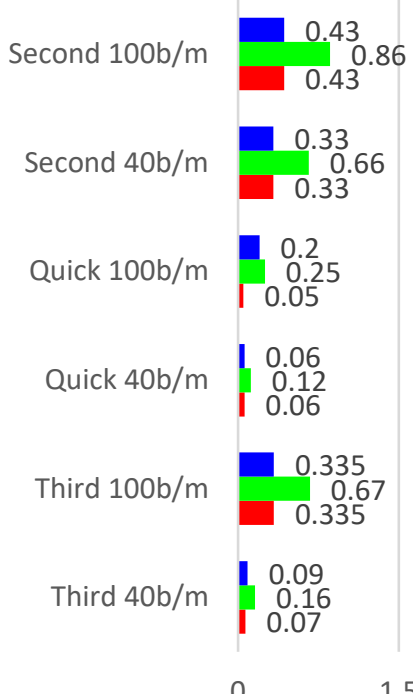
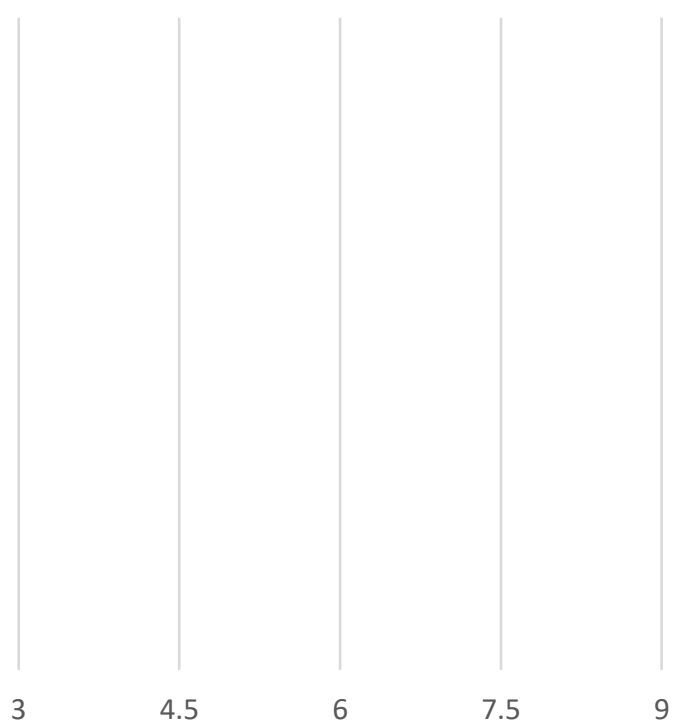

b)
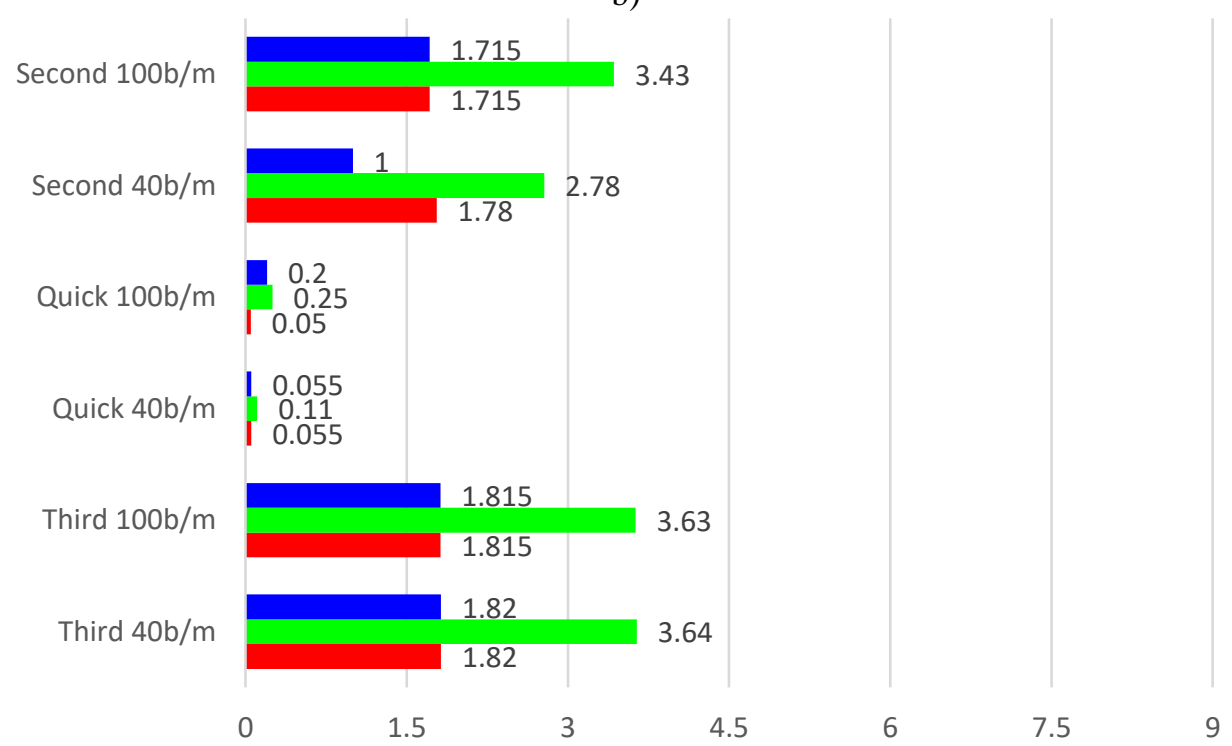

Fig. 6. Hexahedra mesh type, the results of the occurrence of temperatures values outside the input data range of the computation domain in depending on grid mesh density and the computational procedure for Hexahedra mesh type, a) Green-Gauss Node-based, b) Green-Gauss Cell-based. 
a)

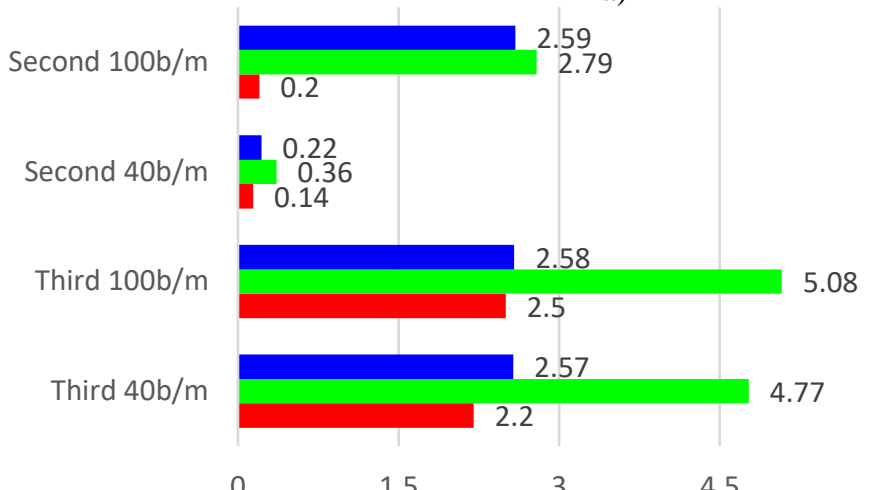

$\begin{array}{lllllll}0 & 1.5 & 3 & 4.5 & 6 & 7.5 & 9\end{array}$

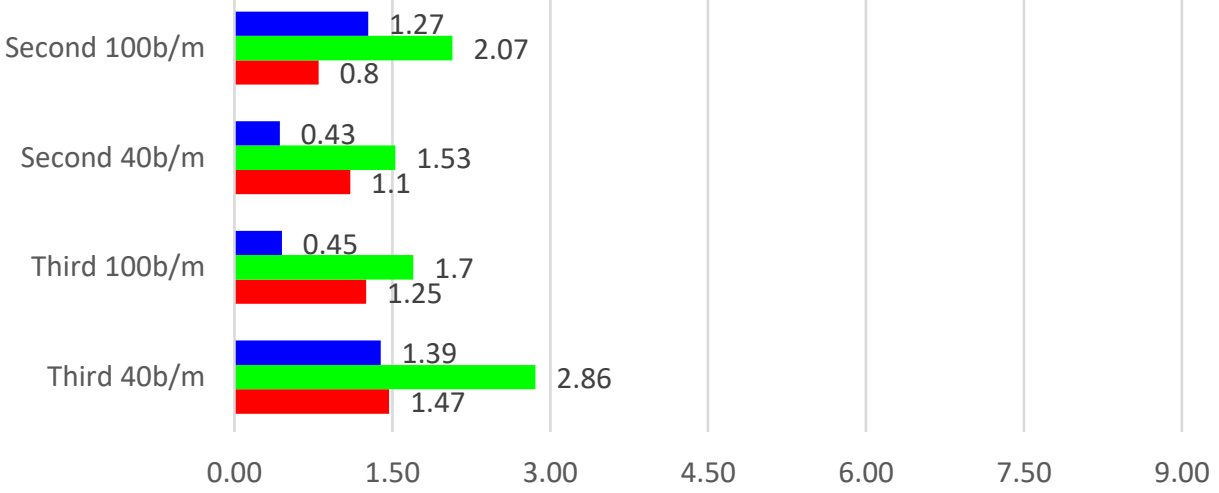

Fig. 7. Tetrahedra mesh type, the temperature values outside the input data range of the computation domain in depending on grid mesh density and the computational procedure for Tetrahedra mesh type, a) Green-Gauss Node-based, b) Green-Gauss Cell-based.

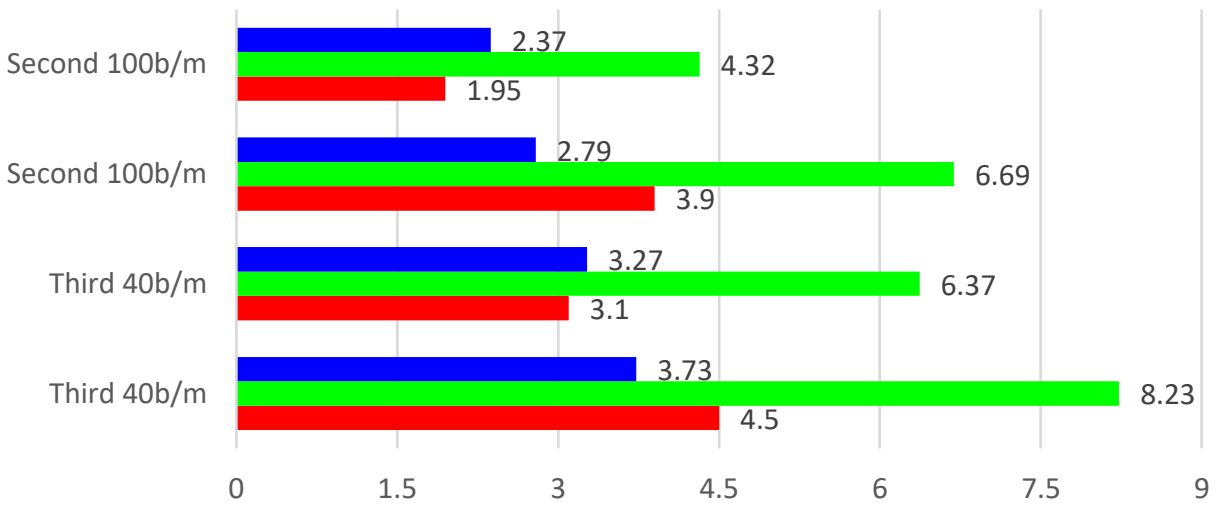

Fig. 8. Polyhedra mesh type, the temperature values outside the input data range of the computation domain in depending on grid mesh density and the computational procedure Least Squares CellBased for Polyhedra mesh type. 


\subsection{Choosing of computation procedure}

The method of solution of the transport variable gradient has a significant influence on the generation of values outside of the range of the input parameters, as well as the choice of computations procedures. The Hex and Tetrahedra cell calculations were solved by using the recommended Green-Gauss Cell-Based and Green-Gauss Node-Based Methods. The better values give Cell-Based method for the Hexahedra cells, on the other side the Node-Based method for the Tetrahedra cells.

The regions with Polyhedra cells were computed only with the Least Sguares Cell-Based method, it is due to recommending of cell in the ANSYS-Fluent Manual. However this method achieves the weakest results, mainly in the height of values from the required parameters, which are well evident in Figs. 6-8.

The results of all the variants of the calculations each other are compared. Fig. 6 presents influence of the grid mesh density, calculation scheme and the computational procedure on the results of the occurrence of temperatures values outside the input data range of the computation domain in the case of using Hexahedra mesh type. Fig. 6a documents the results for Green-Gauss Node-based and Fig. $6 \mathrm{~b}$ for Green-Gauss Cell-based.

The temperature values outside the input data range are presented with blue and red colours, the values bellow than $300 \mathrm{~K}$ by blue colour and the values higher than $400 \mathrm{~K}$ by red colour. The values of the total divergence of transport variable are shown by green colour.

Fig. 7 and 8 present the results of the temperature values outside the input data range of the computation domain in depending on grid mesh density and the computational procedure. Fig. 7 shows the results for the Hexahedra mesh type a) for Green-Gauss Node-based, b) Green-Gauss Cell-based. Fig. 8 documents the results for the Polyhedra mesh type - Least Squares Cell-based. The green/red/blues colours present the total/upper/lower divergence of transport variable.

\section{Conclusion}

This paper presents the numerical experiments and the evaluation of the physical accuracy of the numerical calculation in depending on the choice simulation parameters as: the choice of grid mesh density, the choice of grid mesh type, the choice of calculation scheme and the choice of computation procedure. The overview, the comparing, and the results of all divergence of transport quantity from numerical simulations in which the errors occur are presented in Figs. 6-8.

The best results are obtained using the Hexahedra mesh grid type and the Quick Upwind calculation scheme. In this case, the method of calculating of the gradient has a negligible effect on to the occurrence of values outside the "correct" temperature range. The smaller diffusion occurs with a thicker mesh grid.

The Polyhedra mesh grid gives a smaller number of cells in computation domain, but the relatively high errors of transport variables values, which are outside of the temperature interval.

The choice of computation procedure First-order gives not temperature fields outside of considered interval of temperature.

Financial support from VŠB-Technical University of Ostrava by means of the Czech Ministry of Education, Youth and Sports through the Institutional support for conceptual development of science, research and innovations for the year 2019 and by the Scientific Grant Agency of the Ministry of Education of Slovak Republic and the Slovak Academy of Sciences the project VEGA 1/0374/19 is gratefully acknowledged. 


\section{References}

1. D. P. Karadimou, N. C. Markatos, Numerical Simulations in Enmgineering Science, (2018).

2. V. Michalcová, Procedia Engineering, 190 (2017).

3. G.S. Brar, S. Singh, Procedia Technology, 14 (2014).

4. H. K. Versteeg, W Malalakera, W. Malalasekera, An Introduction to Computational Fluid Dynamics, (2007).

5. F. T. Dodge, The new "dynamic behavior of liquids in moving containers". 2000.

6. K. Kotrasová, Advanced Materials Research, 969 (2014).

7. M. Kozubková, T. Blejchar, M. Bojko, Modeling of heat, mass and momentum transfer (2019).

8. D. Foti, Structural Control and Health Monitoring, 26 (2019).

9. J. Melcer, MATEC Web of Conferences, 107 (2017).

10. I. S. Leoveanu, D. Taus, K. Kotrasova, E. Kormanikova, ACMOS'11, (2011).

11. N. Jendželovský, IOP Conference Series:Material Science and Enfgineering 471 (2019).

12. R. B. Lants, Society of Petroleum Engineers Journal, 11 (1971).

13. M. Krejsa, M, J. Brozovsky, S. Seitl, Z. Kala, V. Krejsa, P. Lehner, ESREL (2018).

14. J. C. Knievel, G. H. Bryan, J. P. Hacker, American Meteorological Society, 135 (2007).

15. K. Kotrasová, I. Grajciar, Advanced Materials Research, 969 (2014).

16. P. Schwartz, D. Adalsteinsson, P. Colella, A. P. Arkin, M. Onsum, PNAS, 102 (2005).

17. V. Michalcova, L. Lausova, I. Kolos, MATEC Web of Conferences, 107 (2017).

18. D. C. Wilcox, Turbulence Modeling for CFD, (2006).

19. M. Major, M. Kosin, I. Major, E3S Web of Conferences, 97 (2019).

20. Q. Chen, J. Srebric, How to Verify, Valide and Report Indoor Enviroment Modelling, (2001).

21. W. L. Oberkampf, T. G. Trucano, Prog. Aerosp. Sci., 38 (2002).

22. J. Liu, E. S. Oran, C. R. Kaplan, Journal of Computational Physics, 108 (2005).

23. J. Králik, J. Králik, AIP Conference Proceedings, 1978 (2018).

24. Q. Chen, J. Srebric, Int. J. HVAC\&R Res., 8 (2002).

25. K. Kotrasová, Procedia Engineering, 190 (2017).

26. M. Močilan, M. Žmindák, P. Pecháč, P. Weis, Procedia Engineering, 192 (2017).

27. K. Zgodavova, M. Mihalikova, S. Hurna, M. Straka, V. Mikloš, Przemysl chemiczny, 97/2 (2018).

28. L. Lapčík, M. Vašina, B. Lapčíková, E. Otyepková, K. E. Waters, Compos Part B Eng 77 (2015).

29. R. Peyret, Computational Fluid Mechanics, (1996).

30. K. Tvrdá, Vibroingineering Procedia, 23 (2019).

31. M. Saqib, S. Hasnain, D. S. Mashat, AIP Advances, 7 (2017).

32. L. Lausová, I. Kološ, V. Michalcová, Civil and Environmental Engineering, 15/1 (2019). 